\title{
EHMTI-0204. Efficacy of SPG stimulation in relieving acute cluster pain: results from $>5000$ attacks treated during long-term follow-up of the pathway $\mathrm{CH}-1$ study
}

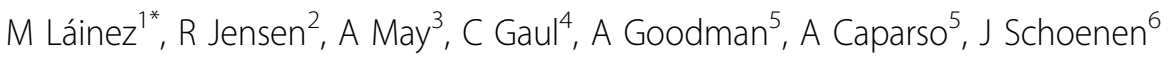 \\ From 4th European Headache and Migraine Trust International Congress: EHMTIC 2014 \\ Copenhagen, Denmark. 18-21 September 2014
}

\section{Introduction}

In the randomized, double-blind, multi-center study of an SPG neurostimulator (Pathway $\mathrm{CH}-1$ study) $68 \%$ of patients experienced clinically significant improvements.

\section{Aim}

The aim of this interim analysis is to evaluate acute response of SPG stimulation therapy for the treatment of $\mathrm{CCH}$ during Long-Term Follow-Up (LTFU).

\section{Method}

43 patients with refractory $\mathrm{CCH}$ (minimum 4 attacks/ week) were enrolled in the Pathway CH-1 study; 33 continued into LTFU (from an average of 14 months through up to 3 years following neurostimulator insertion). Each attack treated with SPG stimulation therapy was evaluated for effective therapy (relief from moderate or greater pain, or freedom from mild pain). All evaluable attacks during the LTFU period through March 2014 were included in the analysis.

\section{Results}

Twenty-six patients provided data for treatment of at least one cluster attack during LTFU. Time in LTFU was an average of 365 days (range 117-575). Average number of attacks treated per patient was 197 (range 1-1489). A total of 5132 attacks were treated (22\% mild initial pain, $47 \%$ moderate, $22 \%$ severe, $9 \%$ very severe). $65 \%(\mathrm{~N}=3354$ / 5132) of these attacks achieved effective therapy (59\% of mild attacks, $78 \%$ of moderate, $62 \%$ of severe, $21 \%$ of very severe). Average stimulation duration was 12.9 minutes.

\section{Conclusion}

Two-thirds of the more than 5000 cluster attacks evaluated during the LTFU period are effectively treated with SPG stimulation therapy.

No conflict of interest.
Authors' details

${ }^{1}$ Neurology, Hospital Clinico Universitario, Valencia, Spain. ${ }^{2}$ Neurology, Danish Headache Society, Glostrup, Denmark. ${ }^{3}$ Neurology, Universitätsklinikum Hamburg-Eppendorf, Hamburg, Germany. ${ }^{4}$ Neurology, University DuisburgEssen, Essen, Germany. ${ }^{5}$ Clinical, Autonomic Technologies Inc., Redwood City, USA. ${ }^{6}$ Neurology, CHR de la Citadelle, Liège, Belgium.

Published: 18 September 2014

doi:10.1186/1129-2377-15-S1-C22

Cite this article as: Láinez et al:: EHMTI-0204. Efficacy of SPG stimulation in relieving acute cluster pain: results from $>5000$ attacks treated during long-term follow-up of the pathway $\mathrm{CH}-1$ study. The Journal of Headache and Pain 2014 15(Suppl 1):C22.

${ }^{1}$ Neurology, Hospital Clinico Universitario, Valencia, Spain

Full list of author information is available at the end of the article

C 2014 Láinez et al; licensee Springer. This is an Open Access article distributed under the terms of the Creative Commons Attribution License (http://creativecommons.org/licenses/by/2.0), which permits unrestricted use, distribution, and reproduction in any medium, provided the original work is properly cited. 